${ }^{2}$ Klinik für Neurologie, Städtisches Klinikum Karlsruhe gGmbH, Karlsruhe, Deutschland

\title{
Facharztstandard und Leitlinien im Arzthaftungsrecht
}

Medizinisch-wissenschaftliche Leitlinien von Fachgesellschaften, nationale Versorgungsleitlinien und onkologische Leitlinien spielen in der ärztlichen Praxis eine zunehmend größere Rolle und sind auch aus dem medizinrechtlichen Alltag nicht mehr wegzudenken. Leitlinien sollen den medizinischwissenschaftlichen Erkenntnisstand im Zeitpunkt ihrer Erstellung bündeln und abbilden. Es handelt sich dabei um Hilfen bei der Entscheidungsfindung in spezifischen Behandlungssituationen, die jedoch keinen rechtsverbindlichen Charakter haben. Ist eine ärztliche Handlung nicht leitlinienkonform, kann daher hieraus nicht automatisch eine Standardunterschreitung hergeleitet werden. Und umgekehrt befreit das Befolgen einer Leitlinienempfehlung im Einzelfall nicht automatisch vom Vorwurf eines Behandlungsfehlers. Dies hat das Oberlandesgericht (OLG) Köln in einer beachtenswerten Entscheidung noch einmal bestätigt (OLG Köln, Urteil vom 15.10.2018, AZ: $5 \mathrm{U}$ $76 / 16)$.

\section{Der Fall}

In dem Rechtsstreit ging es u.a. um Schmerzensgeldansprüche wegen des Vorwurfs einer nicht leitliniengerechten Vorgehensweise im Rahmen einer laparoskopischen Blinddarmentfernung. Der Operateur hatte die Trokaröffnung von $12 \mathrm{~mm}$ nicht durch eine Fasziennaht verschlossen. Die adipöse Patientin erlitt später eine Trokarhernie, wodurch es zur Einklemmung und Perforation des Darms kam. Die Patientin verstarb schließlich infolge eines septischen Schocks. Die zum Zeitpunkt der Behandlung geltende Leitlinie enthielt die Empfehlung, bei Trokarzugängen von mehr als $10 \mathrm{~mm}$ auch die Faszie zu verschließen.

\section{Die Entscheidung}

Das Oberlandesgericht Köln wies die Klage in zweiter Instanz vollständig ab, da im Rahmen der Beweisaufnahme kein Behandlungsfehler festgestellt werden konnte. Vielmehr sei der fachärztliche Standard eingehalten worden. Denn der Begriff des Standards - so das OLG wörtlich - „gibt im Arzthaftungsrecht Auskunft darüber, welches Verhalten von einem gewissenhaften und aufmerksamen Arzt in der konkreten Behandlungssi-

\section{Redaktion \\ G. Gahn, Karlsruhe \\ A. Wienke, Köln}

DGNeurologie 2020 · 3 (6): 565-567

https://doi.org/10.1007/s42451-020-00188-4

Online publiziert: 4. Mai 2020

(c) Der/die Autor(en) 2020 tuation aus der berufsfachlichen Sicht seines Fachbereichs im Zeitpunkt der Behandlung vorausgesetzt und erwartet werden kann. Er repräsentiert den jeweiligen Stand der naturwissenschaftlichen Erkenntnisse und der ärztlichen Erfahrung, der zur Erreichung des ärztlichen Behandlungsziels erforderlich ist und sich in der Erprobung bewährt hat. "Auch der Bundesgerichtshof nahm in vorangegangenen Verfahren mit dieser Argumentation eine Abgrenzung der Ziele und Aufgaben medizinischer Leitlinien vom rechtlich bedeutsamen Standard vor (vgl. z. B. BGH Urteil vom 24.02.2015, AZ: VI ZR 106/13).

Der vom OLG Köln beauftragte ärztliche Sachverständige hatte im Rahmen der Beweisaufnahme erläutert, dass etwa die Hälfte der Operateure im Zeitpunkt der damaligen Operation die Trokaröffnung grundsätzlich nicht oder nur in hier nicht vorliegenden Ausnahmefällen verschlossen habe. Die Versorgung von Trokarzugängen sei zum damaligen Zeitpunkt umstritten gewesen. Dazu komme, dass damals ein bedeutender Teil der Ärzte auch in vergleichbaren Fällen aus verschiedenen medizinischen Gründen von der Leitlinie abgewichen sei.

Aufgrund dieser Bewertungen des Sachverständigen kam das OLG Köln zu der Überzeugung, dass in dem Nichtverschließen der $12 \mathrm{~mm}$ großen Trokaröffnung jedenfalls im Behandlungszeitpunkt keine Standardunterschreitung vorgelegen habe, auch wenn diese Vorgehensweise nicht den Empfehlungen der Leitlinie folgte. Denn wenn ein großer Teil der Ärzteschaft damals noch anders vorginge, als es die Leitlinie vorsehe, könne nicht davon ausgegangen werden, dass die Leitlinienempfehlung dem aktuellen und allseits konsentierten Stand der naturwissenschaftlichen Erkenntnis und der ärztlichen Erfahrung entsprach.

\section{Fazit}

Die Entscheidung zeigt, dass ein Verstoß gegen in Leitlinien gefasste Empfehlungen nicht zwingend zu einer Standardunterschreitung und damit zu einem Behandlungsfehler führen muss. Vielmehr ist die konkrete Handhabung in der Praxis für die Bewertung der Einhaltung des Standards maßgeblich. Damit bestätigt das OLG Köln zugleich den Charakter der Leitlinien als fachliche Hilfestellung für Ärzte, ohne dass diese Rechtsverbindlichkeit besitzen. Für die Feststellung des medizinischen Standards im Arzthaftungsprozess bedarf es daher stets mehr als den einfachen Vergleich einer Behandlung mit den Vorgaben der Leitlinie (vgl. BGH Urteil vom 15.04.2014, AZ: VI ZR 382/12). Die Einholung eines qualifizierten Sachverständigengutachtens ist daher unumgänglich, um jedem einzelnen Arzthaftungsprozess gerecht zu werden. 


\section{Ein Beispiel aus der Praxis}

(Georg Gahn, Beschwerdeverfahren vor der Schlichtungsstelle einer Landesärztekammer, anonymisiert und gekürzt).

Ein 76-jähriger Mann, am 03.02.2017 Einweisung durch Hausarzt, seit dem Aufstehen morgens gegen 6:30 Uhr Taubheitsgefühl Bein rechts. 4 Tage vorher plötzlich am Computer Finger rechts nicht mehr richtig gespürt, rechtes Bein weniger gefühlt, im Stuhl nach links gerutscht, Gefühl im Bein und Arm nach $1 \mathrm{~h}$ wieder normal. Aufnahme 12:36 Uhr in zertifiziertem Schlaganfallzentrum. Befund: leichte faziale Mundastschwäche rechts sowie leichte latente Hemiparese auf der rechten Seite. NIHSS 1-2 bei Aufnahme, CCT normal. Aufnahmediagnose: lakunärer Hirninfarkt. Blutdruck 187/100 mm Hg, $12,5 \mathrm{mg}$ Urapidil i.v., um 14:30 Uhr $165 / 107 \mathrm{~mm} \mathrm{Hg}$. ASS $500 \mathrm{mg}$ p.o. EKG: Sinusrhythmus.

\section{Diagnostik}

Karotis-/transkranielle Duplexsonographie vom 04.02.2017, Uhrzeit 12:07 Uhr: keine hämodynamisch relevanten Stenosierungen, leichte Atherosklerose. cMRT/MRA 06.02.2017: fragmentierte links hochparietale Anterior- und Mediaischämien. Stenosen im linken P2 und rechten P1.

Langzeit-EKG: Wechsel zwischen Sinusrhythmus und intermittierender Tachyarrhythmie bei Vorhofflimmern.

Langzeitblutdruck: überwiegend normotone Blutdruckwerte, nachts keine ausreichende Absenkung.

\section{Verlauf}

Patient lehnt stationäre Aufnahme ab. Auf Drängen des Aufnahmearztes in Aufnahme eingewilligt. Stroke-Unit belegt, keine Triagepatienten verlegbar, IMC- und ITS-Kapazitäten ebenfalls belegt. Aufnahme auf neurologische Normalstation (Anordnung 3-mal täglich RR, ASS $100 \mathrm{mg}$, Ramipril und Amlodipin begonnen).

Pflegedokumentation: 03.02.2017, 19:00 Uhr, Beschwerden Arm und Bein wieder tauber. Ärztin sei informiert, schaue die Situation an. 04.02.2017, 11:00 Uhr rechte Seite noch schläfrig. Samstag weitere Verschlechterung der rechtsseitigen Halbsymptomatik, keine Konsequenzen.

Ärztliche Dokumentation: 03.02.2017 Amlodipin $5 \mathrm{mg}$. 04.02.2017 RR-Messung $110 / 80 \mathrm{~mm} \mathrm{Hg}$ (3-mal täglich angeordnet), Jonosteril abgesetzt. 06.02.2017 schwere motorische Aphasie, Neglect nach rechts, schwere Armparese rechts, Bein auf Unterlage bewegt, Fallneigung nach rechts im Sitzen, Kontrolle zerebrale Bildgebung bei progressivem Schlaganfall.

\section{Vorwurf des Patienten}

Er hätte auf der Stroke-Unit aufgenommen werden müssen. Dann hätte man seinen schweren Schlaganfall verhindern können.

\section{Stellungnahme des Gutachters}

(Hinweis: Es handelt sich um ein Schlichtungsverfahren und nicht um ein Arzthaftungsverfahren in einem Zivilprozess):

Der Patient bemerkte am Aufnahmetag morgens eine rechtsseitige leichte Lähmung mit Taubheitsgefühl, welche in ähnlicher Weise 4 Tage zuvor für die Dauer von etwa $1 \mathrm{~h}$ aufgetreten war. Zum Zeitpunkt der Vorstellung in der zentralen Notaufnahme war er klinisch nur leicht betroffen und befand sich außerhalb eines sog. Lysezeitfensters $(>4,5 \mathrm{~h}$ nach Beginn der Symptome).

Dieser Zustand hätte zu einer Aufnahme auf eine StrokeUnit führen sollen.

Eine Einschätzung nach einem Risikoscore für eine mögliche Verschlechterung der neurologischen Symptomatik, z.B. den ABCD2-Score, erfolgte nicht. Danach hätte der Patient mit den Risikofaktoren A (Alter) mit 76 Jahren 1 Punkt erhalten, für den hohen Blutdruck ebenfalls 1 Punkt, für die Art der Symptome 1 Punkt, für die Dauer der Symptome 2 Punkte, für Diabetes 0 Punkte, sodass er insgesamt 5 Punkte erhalten hätte und somit stationär auf einer Stroke-Unit hätte überwacht werden müssen. Dieses Vorgehen hätte den zum damaligen Zeitpunkt gültigen Leitlinien der Deutsche Gesellschaft für Neurologie zur Behandlung des akuten ischämischen Schlaganfalls entsprochen.

„Bei Patienten mit fluktuierender oder nur gering ausgeprägten neurologischen Symptomen (z. B. NIHSS <4, „minor stroke") ist gleichermaßen eine sofortige und vollständige diagnostische Klärung mit dem möglichen Ziel einer systemischen Thrombolyse notwendig. Eine Behandlung auf einer StrokeUnit ist insbesondere bei Risikoindikatoren (z. B. ABCD2 $\geq 4$ oder Vorliegen einer Emboliequelle (z. B. Vorhofflimmern, hochgradige Karotisstenose)) geboten“.

\section{Schlichtungsvorschlag}

Der Patient hätte nach gültigen Leitlinien als

- akuter Schlaganfallpatient eingeschätzt,

- auf einer Stroke-Unit überwacht sowie

- aufgrund der am Abend beschriebenen Verschlechterung sofort ärztlich untersucht werden müssen.

Aus Kapazitätsgründen konnte der Patient nicht auf einer Stroke-Unit, sondern nur auf einer neurologischen Normalstation aufgenommen werden. Somit wurde er formal nicht entsprechend den zum damaligen Zeitpunkt gültigen Leitlinien behandelt. Auf der Normalstation wurde nicht auf die fortschreitende Verschlechterung des neurologischen Zustandes reagiert.

Ursache des ischämischen Schlaganfalls waren embolische Gefäßverschlüsse im mittleren und vorderen Stromgebiet (Media- und Anteriorstromgebiet). Aufgrund fehlender therapeutischer alternativer Möglichkeiten hätte auch auf einer Stroke-Unit mit entsprechenden Überwachungsmöglichkeiten der Verlauf nicht aufgehalten werden können, sondern wäre schicksalhaft erfolgt. Die von dem Patienten am Morgen des 03.02.2017 bemerkte halbseitige Lähmung hätte auch bei früherer Wahrnehmung seitens der behandelnden Neurologen nicht zu einer Änderung des Therapieregimes führen können. Eine systemische Thrombolyse wäre aufgrund des vorgerückten Zeitfensters kontraindiziert gewesen, dies war bereits am Freitagmorgen am 02.02.2017 abgelaufen. Auch bestanden keine Gefäßveränderungen, die man etwa durch eine Katheterbehandlung oder eine operative Behandlung im Bereich der hirnversorgenden Gefäße hätte behandeln können.

\footnotetext{
1 Leitlinie 030-117 Stand 31.12.2016, gültig bis 30.12.2021, Hennerici M. G., Kern R et al. S1-Leitlinie Diagnostik akuter zerebrovaskulärer Erkrankungen. 2017. In: Deutsche Gesellschaft für Neurologie, Hrsg. Leitlinien für Diagnostik und Therapie in derNeurologie (abgerufen am 23.04.2020).
} 
Eine Verlegung in eine andere Klinik aufgrund der fehlenden Stroke-Unit-Kapazitäten am 03.02.2017 wäre sicher möglich, aber nicht gerechtfertigt gewesen. Der Patient war zum damaligen Zeitpunkt nur leicht betroffen und zeigte keine Hinweise auf ein rasches Fortschreiten der Symptome. Hier hätte nur eine strukturierte prästationäre Organisation des Rettungsdienstes weiterhelfen können, etwa ein IVENA-System, das aber nicht vorhanden war.

Anmerkung: In einem Zivilprozess hätte der Facharztstandard zunächst allein aus der Ex-ante-Sicht (vorausblickend und nicht rückblickend) bewertet werden müssen. Eine Behandlung nach Facharztstandard und medizinischen Kriterien ist sicher nicht erfolgt. Die beschriebene Kasuistik veranschaulicht den oft auftretenden Konflikt zwischen logistischen oder strukturellen Problemen und der strikten Befolgung von Leitlinien. Je nach Wertung dieses Konfliktes durch das Gericht hätte die Frage nach einem (groben) Behandlungsfehler auch zu einer Beweislastumkehr und einer anderen Bewertung des Behandlungsverlaufs führen können.

Die Frage nach dem Befolgen einer Leitlinienempfehlung im Einzelfall ist brandaktuell, insbesondere angesichts der aktuellen Diskussion um eine Triagierung von COVID-19-Patienten mit einem Konflikt zwischen leitliniengerechtem Behandeln und begrenzter Verfügbarkeit von Behandlungsmöglichkeiten.

\section{Korrespondenzadresse}

\section{Dr. A. Wienke}

Wienke \& Becker - Köln

Sachsenring 6, 50677 Köln, Deutschland

awienke@kanzlei-wbk.de

\section{Einhaltung ethischer Richtlinien}

Interessenkonflikt. A. Wienke, L. Hübner und G. Gahn geben an, dass kein Interessenkonflikt besteht.

Für diesen Beitrag wurden von den Autoren keine Studien an Menschen oder Tieren durchgeführt. Für die aufgeführten Studien gelten die jeweils dort angegebenen ethischen Richtlinien.

\section{Medizinische Leitlinien sind nicht justiziabel}

Auch das Bundesverwaltungsgericht lässt Ärzten freie Hand

Die Diskussion um Bedeutung, Inhalte und Verbindlichkeit medizinischwissenschaftlicher Leitlinien hält schon seit vielen Jahren an. die Rechtsprechung hat sich verschiedentlich zur rechtlichen Bedeutung und Wirkung von Leitlinien geäußert. Diskutiert wird in diesem Zusammenhang insbesondere die Frage der rechtlichen Verbindlichkeit der Inhalte von Leitlinien.

Der Bundesgerichtshof (BGH) hat bereits wegweisende Entscheidungen getroffen. Auch die Instanzgerichte (s. OLG Köln, Urteil vom 15.10.2018 - in diesem Heft) folgen diesen Entscheidungen. Erstmals hat sich nun auch das Bundesverwaltungsgericht (BVerwG) in seinem Beschluss vom 16.04.2020 - BVerwG 2 B 5.19 - mit der Frage der medizinischen und rechtlichen Verbindlichkeit von Leitlinieninhalten befasst und ausgeführt:

„Leitlinien von ärztlichen Fachgremien oder Verbänden können (im Gegensatz zu den Richtlinien der Bundesausschüsse der Ärzte und Krankenkassen) nicht unbesehen mit dem für die Beurteilung des Gesundheitszustandes gebotenen wissenschaftlichen Standard gleichgesetzt werden. Sie können nicht ohne weiteres als Maßstab für diesen Standard übernommen werden. Die Feststellung des Standards obliegt der Würdigung des sachverständig beratenen Tatsachengerichts (vgl. BGH, Beschluss vom 28. März 2008-VI ZR 57/07)."

Ungeachtet dieser an sich klaren Aussagen der Rechtsprechung zur rechtlichen Einordnung und Zielsetzung werden Leitlinien medizinischwissenschaftlicher Fachgesellschaften zunehmend zur Begründung ärztlichen Fehlverhaltens herangezogen. Der pauschale Vorwurf, von den Empfehlungen einer Leitlinie abgewichen zu sein, ist schnell erhoben. Eine nähere Konkretisierung der angeblichen Behandlungsfehler erfolgt oft nicht. Zudem wird schlicht ins Blaue hinein behauptet, dass bestimmte Komplikationen oder unerwünschte Entwicklungen der Erkrankung bei Beachtung einer bestimmten Leitlinie nicht eingetreten wären. All dies drängt den in das gerichtliche Verfahren involvierten Arzt zur Rechtfertigung seines Handelns oder Unterlassens. Zivilprozessual eine verkehrte Welt: Schließlich muss der Patient darlegen und beweisen, dass die ärztlichen Maßnahmen nicht indiziert oder behandlungsfehlerhaft durchgeführt wurden. Es schleicht sich mit dem Verweis auf die Leitlinien damit eine stille Beweislastumkehr ein, die an sich nur für die Fälle eines sog. groben Behandlungsfehlers anzunehmen ist.

Der Umgang mit medizinisch-wissenschaftlichen Leitlinien muss daher von allen Beteiligten immer sehr zurückhaltend und einzelfallbezogen erfolgen. Die Rechtsprechung hilft den Ärzten und gewährleistet weitgehend freie Hand bei der fachgerechten ärztlichen Diagnostik und Therapie.

Köln im August 2020

Rechtsanwalt Dr. Albrecht Wienke

Wienke \& Becker - Köln

AWienke@kanzlei-wbk.de 